\title{
The Metal Absorption Systems of the FDF QSO 0103-260
}

\author{
Immo Appenzeller, Stefan Noll, Otmar Stahl \\ Landessternwarte Heidelberg, Königstuhl, D69117 Heidelberg, Germany
}

Stephan Frank

Department of Astronomy, OSU, 140 W. 18th Ave., Columbus, $\mathrm{OH}$ 43210, USA

\begin{abstract}
Using the UVES echelle spectrograph at the ESO VLT we obtained high-resolution $(\mathrm{R}=40$ 000) spectra of the QSO 0103-260 in the FORS Deep Field (FDF). In addition to numerous Ly forest lines we identified 16 metal absorption systems with redshifts between $0.97<z<$ 3.36. A comparison of the observed redshift distributions of the metal line systems and of the spectroscopically observed galaxies in the FDF shows that the distribution of metal absorption clouds and of the galaxies along the line of sight to the QSO are well correlated and obviously trace the cosmic structure in the direction of the FDF.
\end{abstract}

\section{Introduction}

The QSO 0103-260 $(\mathrm{B}=20.17, \mathrm{z}=3.365)$ is the brightest of 8 QSOs found in the FORS Deep Field (FDF, Appenzeller et al. 2000, Heidt et al. 2003). It is the only high-redshift QSO in the FDF which is sufficiently bright to allow highresolution spectroscopy for a comparison between the line absorption and the galaxy distribution in the direction of the FDF. Therefore, we observed this QSO using the UVES spectrograph at the ESO VLT UT2 (Kueyen) with a spectral resolution $R=40000$.

\section{The Metal Systems}

The observed spectrum shows in addition to a rich Ly $\alpha$ forest many sharp metal absorption lines. These metal lines could be grouped into 16 systems with different redshifts between $z=0.9737$ and the QSO emission line redshift. Three systems have redshifts very close to that of the QSO. The relative line strengths and the ionization state of the different metal systems were found to differ greatly. However most systems show a high ionization state, as predicted for low-metallicity intergalactic clouds (cf. Rauch et al. 1997).

One of the main objectives of the present study was a comparison between the redshift distribution of the QSO absorption lines with the galaxy redshifts in the FDF. As illustrated by Fig. 1, the galaxy redshifts in the FDF show strong clustering, which is well correlated with the metal absorption line redshift 


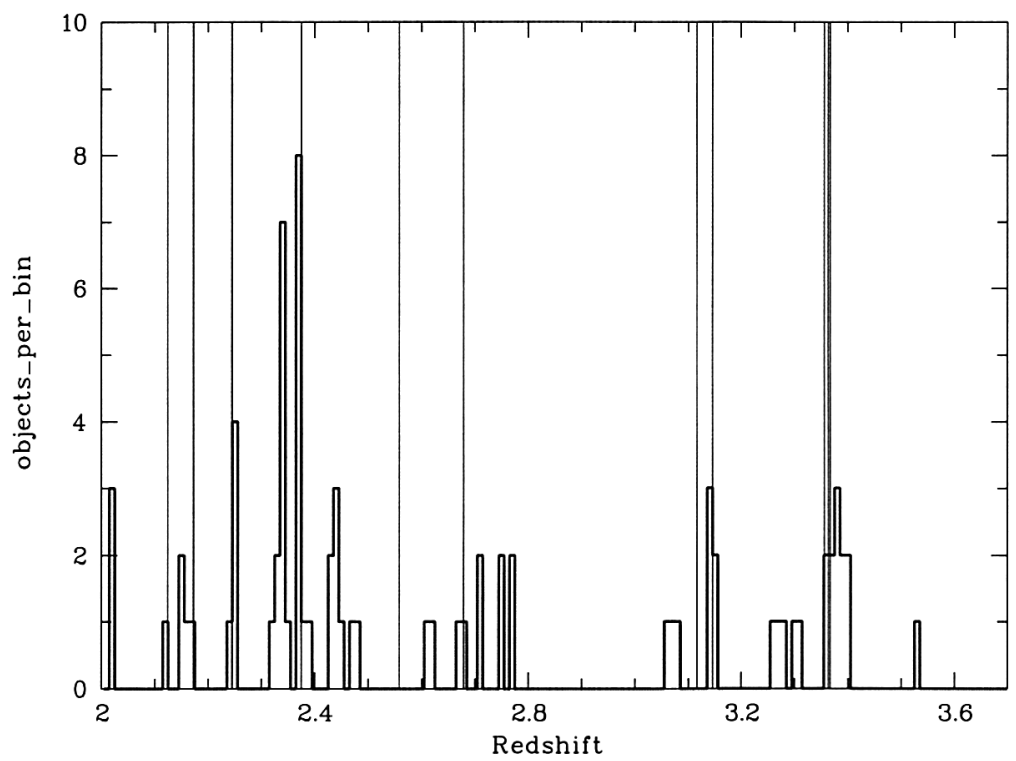

Figure 1. Histogram of the redshift distribution in the spectroscopically observed FDF galaxies in the redshift interval $2.0<\mathrm{z}<3.7$. Also indicated are the redshift positions of the metal absorption systems of Q 0103-260 in this redshift range (thin vertical lines).

distribution. The strength of the metal absorption varies considerably between the different galaxy clusterings and there is at least one significant clustering of galaxies (at $z=2.34$ ) where in spite of a thorough search no metal lines could be detected.

There is also some correlation with the total Ly $\alpha$ absorption. However, our data do not show evidence for a decrease of this absorption in cases where the LOS passes very close to a galaxy. There are again cases where we find HI absorption without metal absorption to coincide with clusterings of the galaxy redshifts, indicating a rather inhomogeneous metal enrichment and/or large variations of the physical conditions of the absorbing gas.

The clustering scale of the galaxies and of the associated metal absorption clouds agrees well with predictions of cosmic structure formation. A more complete discussion of our results can be found in Frank et al. (2003).

\section{References}

Appenzeller, I., et al. 2000, The Messenger 100, 44

Frank, S., et al. 2003, A\&A, 407, 473

Heidt, J., et al. 2003, A\&A 398, 49

Rauch, M., et al. 1997, ApJ 481, 601 